\title{
Antimicrobial Resistance of Enterococcus Species Isolated from Urine Cultures
}

\author{
Mustafa Kerem Çalgın ${ }^{1}$, Yeliz Çetinkol ${ }^{1}$ \\ ${ }^{1}$ Medical Microbiology Department, Ordu University Faculty of Medicine, Ordu, Turkey.
}

Received: 28 June 2019, Accepted: 11 July 2019, Published online: 28 August 2019

(C) Ordu University Institute of Health Sciences, Turkey, 2019

\begin{abstract}
Objective: Enterococcus species are among the common causes of nosocomial urinary tract infections mainly, along with other infections and have been observed to have increasing rates of resistance against antimicrobial agents in recent years. The aim of this study is to determine the Enterococcus spp. isolated from urine cultures in our hospital and to determine antibiotic resistance rates.

Methods: Enterococcus isolates identification and antibiogram results obtained from urine samples sent to Ordu University Education and Research Hospital Microbiology laboratory from inpatients and outpatients were retrospectively evaluated. The identification and antimicrobial susceptibility tests of the isolates were completed on a VITEK 2 Compact (Biomerieux, Marcy l'Etoile, France) system. Resistance rates against ampicillin, ciprofloxacin, nitrofurantoin, tigecycline, linezolid, teicoplanin and vancomycin were analyzed.

Results: Our study identified 346 Enterococcus strains. These strains were defined as 195 Enterococcus faecalis (56\%), 127 Enterococcus faecium (37\%) and 24 other enterococci (7\%). The antimicrobials with highest resistance were ciprofloxacin (51\%), ampicillin (42\%), nitrofurantoin (14\%) and tigecycline (1\%), in order, with no resistance encountered for linezolid, teicoplanin and vancomycin.

Conclusion: Glycopeptide resistance were not encountered among enterococci isolated from urine cultures in our hospital, with quinolone resistance at the fore. The results of antimicrobial susceptibility tests are important to select appropriate treatments.
\end{abstract}

Key words: antimicrobial resistance, Enterococcus species, urine culture

Suggested Citation: Calgin MK, Cetinkol Y. Antimicrobial Resistance of Enterococcus Species Isolated from Urine Cultures. Middle Black Sea Journal of Health Science, 2019; 5(2):133-137.

\section{Address for correspondence/reprints:}

Mustafa Kerem Çalgın

Telephone number: +90 (505) 4951766

E-mail: mkcalgin@gmail.com

DOI: $10.19127 / \mathrm{mbsjohs.583149}$

\section{Introduction}

With the reduction in the efficacy of antibiotics, hospitals around the world have seen increasing numbers of infections due to drug-resistant bacteria. Effective treatment of these infections is more difficult, which causes morbidity and mortality in the patient and increasing health care costs. The most common antimicrobial resistant hospital pathogens are Enterococcus faecium, Staphylococcus aureus, Klebsiella pneumoniae, Acetinobacter baumanni, Pseudomonas aeruginosa and Enterobacter species and are called ESKAPE (Rice, 2008). In humans the enterococcus species causing most infections are Enterococcus faecalis $(80-90 \%)$ and one of the two gram positive 
ESKAPE pathogens of Enterococcus faecium (5$10 \%$ ). These bacteria are among the common causes of nosocomial urinary tract infections mainly, along with other infections (Aykut Arca et al., 2009). Enterococcus species carry a range of intrinsic and acquired resistance genes and may transfer these genes to other bacteria (van Harten et al., 2017). Enterococci may be resistant to commonly used antibiotics including ampicillin and vancomycin and currently resistance has begun to be determined against last-chance antibiotics like daptomycin and linezolid (Gonzales et al., 2001; Long et al., 2005; van Harten et al. 2017).

Due to increasing resistance against commonly used antibiotics, note should be taken of culture results for antimicrobial treatment of Enterococcus infections and the regional resistance phenotypes should be considered when deciding on empirical antibiotic treatment until culture results are obtained. This study aimed to identify the resistance status against a variety of antimicrobials of Enterococcus isolates from urine samples in Ordu University Education and Research Hospital microbiology laboratory.

\section{Methods}

From January 2014 to June 2018, enterococci isolates identifications and antibiogram results obtained from urine samples sent to Ordu University Education and Research Hospital microbiology laboratory from inpatients and outpatients were retrospectively evaluated.

Midflow urine samples taken under appropriate conditions from patients with preliminary diagnosis of urinary tract infections were inoculated on the surface of 5\% sheep's blood agar (Salubris, Istanbul, Turkey) using standard loops taking $1 \mu \mathrm{l}$ urine. The media plates were incubated in an aerobic environment for $18-24$ hours at $37^{\circ} \mathrm{C}$ and cultures with single type proliferation and colony account $10^{5} \mathrm{CFU} / \mathrm{ml}$ were taken for investigation. The identification and antimicrobial susceptibility tests of the obtained isolates were completed in line with Clinical and Laboratory Standards Institute (CLSI) until January 2017 and then in line with the European Committee on Antimicrobial Susceptibility Testing (EUCAST) recommendations on a VITEK 2 Compact (Biomerieux, Marcy l'Etoile, France) system. Resistance rates against ampicillin, ciprofloxacin, nitrofurantoin, tigecycline, linezolid, teicoplanin and vancomycin were analyzed. Strains with intermediate resistance were accepted as resistant. Only one strain from each patient was included in the study. For quality control, the E. faecalis ATCC 29212 standard strain was used.

\section{Results}

Our study identified 346 enterococci strains. These strains were identified as 195 E. faecalis (56\%), 127 E. faecium (37\%) and 24 other enterococci $(7 \%) \quad(9$ E. gallinarum, $7 \quad E$. casseliflavus, 3 E. avium, 3 E. raffinosus, 2 E. durans).

The E. faecalis strains were most common among outpatients, while E. faecium strains were isolated mainly from the intensive care units, with the clinical distribution of these strains given in Table 1.

Table 1. Distribution of E.faecalis and E.faecium strains according to clinics

\begin{tabular}{lcc}
\hline Clinics & E.faecalis & E.faecium \\
\hline Polyclinics & 149 & 42 \\
Intensive Care Units & 20 & 51 \\
Internal medicine Services & 17 & 22 \\
Surgical Services & 6 & 7 \\
Pediatric Services & 3 & 5 \\
\hline Total & 195 & 127 \\
\hline
\end{tabular}

The antimicrobial resistance rates for the isolated E. faecalis and E. faecium strains are shown in Table 2. 
Table 2: Resistance rates of E.faecalis and E.faecium strains

\begin{tabular}{lccccccccc}
\hline & \multicolumn{3}{c}{ E.faecalis } & \multicolumn{3}{c}{ E.faecium } & \multicolumn{3}{c}{ Total* } \\
\cline { 2 - 12 } & $\begin{array}{c}\text { Number of } \\
\text { strains }\end{array}$ & \multicolumn{2}{c}{ Resistant } & $\begin{array}{c}\text { Number of } \\
\text { strains }\end{array}$ & Resistant & $\begin{array}{c}\text { Number of } \\
\text { strains }\end{array}$ & Resistant \\
\cline { 2 - 12 } Antibiotic & $\mathrm{n}$ & $\mathrm{n}$ & $\%$ & $\mathrm{n}$ & $\mathrm{n}$ & $\%$ & $\mathrm{n}$ & $\mathrm{n}$ & $\%$ \\
\hline Ampicillin & 170 & - & 0 & 124 & 124 & 100 & 294 & 124 & 42 \\
Ciprofloxacin & 184 & 56 & 30 & 114 & 95 & 83 & 298 & 151 & 51 \\
Tigecycline & 182 & - & 0 & 103 & 3 & 3 & 285 & 3 & 1 \\
Nitrofurantoin & 36 & 3 & 8 & 15 & 4 & 27 & 51 & 7 & 14 \\
Linezolid & 192 & - & 0 & 122 & - & 0 & 314 & - & 0 \\
Teicoplanin & 184 & - & 0 & 124 & - & 0 & 308 & - & 0 \\
Vancomycin & 191 & - & 0 & 121 & - & 0 & 312 & - & 0 \\
\hline
\end{tabular}

$\mathrm{n}$ :Number, \%:Percent, * Sum of E.faecalis and E.faecium

\section{Discussion}

Enterococci are bacteria forming the normal flora in the gastrointestinal system, vagina and urethra of humans but may also cause a variety of infections. They may survive for long periods on inorganic material like stethoscopes, door handles, and beds in the hospital environment. As a result, enterococci may cause epidemics as a hospital infection vector carried on both inorganic materials and from patient to patient by health personnel (Butler, 2006). In recent years, the observation of an increase in vancomycin resistant strains in isolation and the variation in antibiotic susceptibility according to species have led to the requirement for species level identification. Studies about the topic have shown that in urine samples generally $E$. faecalis isolation rates are higher compared to $E$. faecium (Yuksel Ergin et al., 2013; Etiz et al., 2014; Yenisehirli et al., 2016). In our study the results were similar with $56 \%$ E. faecalis and $37 \%$ E. faecium identification rates.

Compared to other Enterococcus species, $E$. faecalis is found at higher rates in feces. Epidemiologic studies in recent years have shown that the presence of these bacteria in normal intestinal flora is a basic risk factor for the spread of enterococci from patient to patient and even between hospitals (Butler, 2006). Some studies have found higher rates of $E$. faecium strains among enterococci from hospital isolates compared to $E$. faecalis (Aykut Arca et al., 2009). In our study, E. faecalis was isolated more from outpatients, while E. faecium was isolated more from inpatients. This situation may be linked to the ability of E. faecium, found at high rates in intestinal microbiome, to spread between patients.

Treatment of Enterococcus infections has become complicated since the emergence of strains with high levels of resistance to nearly all antibiotics used in clinical practice, especially aminoglycosides, $\beta$-lactams and glycopeptides. Enterococci are the most common vector for infections of the urinary tract. Enterococci are known to be more resistant to antimicrobials affecting inhibition of cell wall synthesis compared to other streptococci and the use of penicillin or ampicillin is recommended for susceptibility tests (Murray, 1997). Studies in Turkey have noted that enterococci are increasingly resistant to beta-lactam antibiotics and a variety of studies have reported ampicillin resistance from $16 \%$ to $84 \%$ (Baykan, 2001; Agus et al., 2006; Aktepe et al., 2011; Kalayci et al., 2011; Yuksel Ergin et al., 2013; Etiz et al., 2014). In our study, rates of $42 \%$ were between these two values.

Quinolones are found to have limited efficacy against enterococci. As a result, though they are effective in vitro, their use for treatment of infections caused by these bacteria is limited (Gordon et al., 1992). Ciprofloxacin is approved for use for both uncomplicated and complicated urinary tract infections, including cystitis, pyelonephritis, and chronic bacterial prostatitis (Andriole, 2005). Among enterococci infections, they are among alternative treatment choices only for urinary tract infections (Gordon et al., 1992). A variety of studies have identified ciprofloxacin resistance from $48 \%$ to 87\% (Baykan, 2001; Yavuz et al., 2006; Aktepe et al., 2011; Kalayci et al., 2011; Yuksel Ergin et al., 2013; Etiz et al., 2014). In our study, ciprofloxacin resistance was close to the lower limit at $51 \%$.

Tigecycline is an antibiotic derived from the first member of the glycylcyclines of minocycline and a promising new antibiotic of last resort, active against many bacteria including Enterococcus spp. (Tunger, 2012). Studies by Karaoglan et al. and 
Aktepe et al. did not report resistance for enterococci against tigecycline, while Etiz et al. reported $0.3 \%$ resistance (Karaoglan et al., 2008; Aktepe et al., 2011; Etiz et al., 2014). In our study, Enterococcus spp. resistance to tigecycline was $1 \%$, and all resistant isolates were observed to be $E$. faecium. Nitrofurantoin may be used for uncomplicated urinary tract infections (Tunger, 2012). The use of nitrofurantoin with appropriate indications will reduce the use of new antimicrobials and as a result their risk of resistance developing. In our study, $14 \%$ rates of resistance against nitrofurantoin were encountered and this resistance was observed more for the E. faecium isolates.

Glycopeptides are still known as the most effective antibiotics against enterococci, with increased rates of vancomycin and teicoplanin resistant strains reported (Agus et al., 2006; Yuksel Ergin et al., 2013; Etiz et al., 2014). In this study, vancomycin and teicoplanin resistance was not encountered among E. faecalis and E. faecium isolates.

Linezolid is an oxazolidinone group antibiotic effective against many gram positive bacteria including vancomycin-resistant enterococci (VRE) (Dilek et al., 2007). It is recommended for treatment of infections caused by VRE (Contreras et al., 2019). In our study, similar to many studies performed to date, linezolid resistance was not encountered among enterococci (Dilek et al., 2007; Aktepe et al., 2011; Yuksel Ergin et al., 2013). Contrary to this, Etiz et al. in a 2014 study found 5\% resistance to linezolid and reported the necessity to use this agent with appropriate indications and sufficient doses by performing antibiotic susceptibility tests, as for other antimicrobials to prevent development of resistance (Etiz et al., 2014).

\section{Conclusion}

Enterococci have become microorganisms threatening health and causing problems with treatment today. They are resistant to many drugs and the increase in this resistance will continue to increase problems like causing difficult clinical infections. As a result, as for all infection agents, rational antibiotic use is necessary for treatment of infections linked to Enterococcus species.
Ethics Committee Approval: Ethics committee approval was not received because it was a retrospective study.

Peer-review: Externally peer-reviewed.

Author Contributions: Externally peer-reviewed. Author Contributions: Concept- MKC, YC; Design- MKC; Supervision- MKC, YC; FundingMKC, YC; Materials- MKC, YC; Data Collection/Data Processing- MKC; Analyze and Interpretation- MKC; Literature Review-MKC; Writing- MKC; Critical Review- MKC, YC.

Conflict of Interest: No conflict of interest was declared by the author.

Financial Disclosure: The authors declared that this study hasn't received no financial support.

\section{References}

Agus N, Sarica A, Ozkalay N, Cengiz A. Antimicrobial Resistance of Enterococcus Strains Isolated from Clinical Specimens. ANKEM J. 2006;20(3):145-7.

Aktepe OC, Asik G, Ciftci IH, Cetinkaya Z. Antibiotic Resistance Rates in Enterococcus Strains Isolated from Clinical Specimens. Turk Mikrobiyol Cem J 2011;41(2):86-90.

Andriole VT. The Quinolones: Past, Present, and Future. Clinical Infectious Diseases. 2005;41(Supplement_2):113-9.

Aykut Arca E, Mert Dinc B, Karabiber N. Distribution to Clinics of Enterococci Species Isolated from Various Clinical Samples. Turkish Bulletin of Hygiene and Experimental Biology. 2009;66(1):1-5.

Baykan M. Evaluation of invitro antibiotic sensitivity of Enterococcus isolated from urine samples. Gen Med J. 2001;11(3):119-21.

Butler KM. Enterococcal Infection in Children. Seminars in Pediatric Infectious Disease: 2006;17(3):128-39.

Contreras GA, Munita JM, Arias CA. Novel Strategies for the Management of VancomycinResistant Enterococcal Infections. Curr Infect Dis Rep. 2019;21(7):22.

Dilek AR, Yildiz F, Dilek N, Bulut Y, Asci Toraman Z. In-vitro Activity of Linezolid Against Methicillin-resistant Staphylococcus aureus (MRSA) and Enterococcus spp. ANKEM J. 2007;21(4): 211-3.

Etiz P, Kibar F, Ekenoglu Y, Yaman A. Evaluation of the Antibiotic Resistance Profiles of Enterococcus Species Isolated from Urine Cultures. Turk Mikrobiyol Cem Derg. 2014;44(3):107-13. 
Gonzales RD, Schreckenberger PC, Graham MB, Kelkar S, DenBesten K, Quinn JP. Infections due to vancomycin-resistant Enterococcus faecium resistant to linezolid. Lancet. 2001;357(9263):1179.

Gordon S, Swenson JM, Hill BC, Pigott NE, Facklam RR, Cooksey RC et al. Antimicrobial susceptibility patterns of common and unusual species of enterococci causing infections in the United States. Enterococcal Study Group. J Clin Microbiol. 1992;30(9):2373-8.

Kalayci O, Yurtsever SG, Gungor S, Uzun B, Kurultay N. Evaluation of In Vitro Antibiotic Sensitivity of Enterococci Isolated from Urine Samples. Klimik J. 2011;24(2):105-7.

Karaoglan I, Zer Y, Namiduru M. In-vitro Activity of Tygecycline for Vancomisin-resistant Enterococcus Strains. ANKEM J. 2008;22(3):153-5.

Long JK, Choueiri TK, Hall GS, Avery RK, Sekeres MA. Daptomycin-resistant Enterococcus faecium in a patient with acute myeloid leukemia. Mayo Clin Proc. 2005;80(9):1215-6.

Murray BE. Vancomycin-resistant enterococci. Am J Med. 1997;102(3):284-93.

Rice LB. Federal funding for the study of antimicrobial resistance in nosocomial pathogens: No ESKAPE. Journal of Infectious Diseases. 2008;197(8):1079-81.

Tunger O. The Old and New Treatment Options for Vancomycin-resistant Enterococcal Infections. ANKEM J. 2012;26(4):215-27.

van Harten RM, Willems RJL, Martin NI, Hendrickx APA. Multidrug-Resistant Enterococcal Infections: New Compounds, Novel Antimicrobial Therapies? Trends Microbiol. 2017;25(6):467-79.

Yavuz MT, Sahin I, Ozturk E, Behcet M, Kaya D. Insidence and antibiyotic resistance profiles of Enterococcus species isolated from nosocomial urinary tract infections. Turk Mikrobiyol Cem Derg. 2006;36(4):195-9.

Yenisehirli G, Yenisehirli A, Bulut Y, Ozveren G. Antimicrobial Resistance of Enterococci Isolated From Urine Cultures. Klimik J. 2016;29(3):112-6.

Yuksel Ergin O, Bayram ED, Uzun B, Gungor S, Demiral T. Enterococcus Species Isolated from Urine Cultures and Their Antibiotic Resistance. ANKEM J. 2013;27(4):173-8. 